SMI-94-1

January, 1994

\title{
Loop Equations as a Generalized Virasoro Constraints
}

\author{
K. Zarembot \\ Steklov Mathematical Institute, \\ Vavilov st. 42, GSP-1, 117966 Moscow, RF
}

\begin{abstract}
The loop equations in the $U(N)$ lattice gauge theory are represented in the form of constraints imposed on a generating functional for the Wilson loop correlators. These constraints form a closed algebra with respect to commutation. This algebra generalizes the Virasoro one, which is known to appear in one-matrix models in the same way. The realization of this algebra in terms of the infinitesimal changes of generators of the loop space is given. The representations on the tensor fields on the loop space, generalizing the integer spin conformal fields, are constructed. The structure constants of the algebra under consideration being independent of the coupling constants, almost all the results are valid in the continuum.
\end{abstract}

\footnotetext{
${ }^{1} \mathrm{E}-$ mail: zarembo@qft.mian.su
} 


\section{Introduction}

The string description of the gauge theories is a classic problem. In particular, the equivalence of the lattice QCD to string theory has been suspected since the pioneer work by Wilson [1]. The arguments are mostly based on the representation of $1 / N$ expansion combined with the strong (or weak) coupling ones in terms of a sum over random surfaces (for a review, e.g. [2]). Recently the substantial progress has been made in the string description of the two dimensional QCD [0].

On the other hand, string theory is usually associated with the existence of high symmetries. It is tempting to understand how do this symmetries emerge in the gauge theories. The useful analogy is provided by the matrix models, connection of which with string theory is well elaborated. The Virasoro algebra emerges in the matrix models as an algebra of Schwinger-Dyson constraints imposed on the partition function (四 and references therein). In this paper we study an algebra that originates in the same way from the loop equations (see [5] for a review) in the lattice gauge theory. Almost all our results remain valid also in the continuum.

\section{The Loop Equations}

Consider the generating functional for Wilson loop correlators

$$
Z[\beta]=\int D U \exp \left(\sum_{C} \beta_{C} \operatorname{tr} U(C)\right),
$$

where the sum is going over all oriented closed contours on the finite lattice and $U(C)$ is the path-ordered product of $U(N)$ valued gauge fields, $U_{x}^{\mu}$, defined on the links. Note that due to unitarity of the link variables we are forced to identify the loops differing by backtrackings (see fig. 1a). The loop correlators can be obtained differentiating $Z$ with

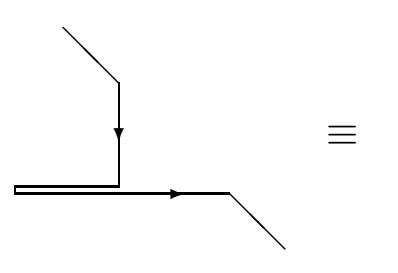

a

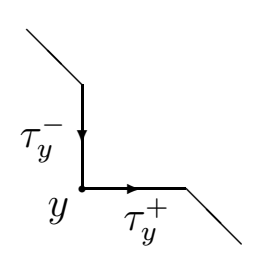

$\mathrm{b}$

Figure 1: a) The backtracking condition. b) The definition of $\tau_{y}^{-}, \tau_{y}^{+}$.

respect to $\beta_{C}$ :

$$
\left\langle\operatorname{tr} U\left(C_{1}\right) \ldots \operatorname{tr} U\left(C_{n}\right)\right\rangle=\frac{1}{Z} \frac{\partial^{n}}{\partial \beta_{C_{1}} \ldots \partial \beta_{C_{n}}} Z
$$


We consider $\beta_{C}$ and $\beta_{C^{-1}}$ as the independent variables, so the action in (2.1) is generally complex. Of course, to calculate the actual values of the loop correlators for a given model one should, after differentiations, put $\beta_{C}$ to their actual values, which satisfy $\beta_{C}=\beta_{C^{-1}}$, say $\beta_{C}=0$ for all $C$ except the single-plaquette couplings $\beta_{\square}=1 / g^{2}$ in the case of the Wilson action for the pure gauge theory. The partition function of the theory with fermions can also be represented in the form (2.1) after integration over the matter fields. The corresponding determinant contributes to the action

$$
\beta_{C}=-k^{|C|} \phi(C) .
$$

There $k$ is a hopping parameter, $|C|$ is a length of the loop $C$ (the number of links) and $\phi(C)$ is a spin factor - the trace of a path-ordered product of gamma matrices for chiral or projectors for Wilson fermions. More generally, the $U(N)$ gauge theory with the arbitrary matter fields in the fundamental representation can be obtained as a reduction of (2.1) to the particular values of $\beta_{C}$, the contribution of each matter field having the form (2.3).

To write down the loop equations for (2.1) it is necessary to introduce the notion of a loop with marked points $-C_{x_{1} \ldots x_{n}}$ (the order of marked points is essential). The loop $C$ may have selfintersections, so the points (or the links), which are different on the loop, may coincide on the lattice. If marked points $x$ and $y$ on the loops $C_{x_{1} \ldots x_{k} x}$ and $D_{y x_{k+1} \ldots x_{n}}$ coincide on the lattice, we can concatenate them to form a loop $C_{x_{1} \ldots x_{k} x} D_{y x_{k+1} \ldots x_{n}}$ with marked points $x_{1}, \ldots, x_{n}$.

Given a loop with one marked point $C_{x}$ and a marked link $l_{x}^{\mu}$ one can derive the loop equation using the invariance of a measure in the integral

$$
\int D U U\left(C_{x}\right) \exp \left(\sum_{C} \beta_{C} \operatorname{tr} U(C)\right)
$$

under an infinitesimal left shift $U_{x}^{\mu} \rightarrow U_{x}^{\mu}+\xi U_{x}^{\mu}$ (see [5] for more details):

$$
\begin{array}{r}
\sum_{D} \beta_{D} \sum_{y \in D} \delta_{y x}\left(\delta_{\tau_{y}^{+}, l_{x}^{\mu}}-\delta_{\tau_{y}^{-},\left(l_{x}^{\mu}\right)^{-1}}\right)\left\langle\operatorname{tr} U\left(C_{x} D_{y}\right)\right\rangle \\
+\sum_{y \in C} \delta_{y x}\left(\delta_{\tau_{y}^{+}, l_{x}^{\mu}}-\delta_{\tau_{y}^{-},\left(l_{x}^{\mu}\right)^{-1}}\right)\left\langle\operatorname{tr} U\left(C_{[x y]}\right) \operatorname{tr} U\left(C_{[y x]}\right)\right\rangle=0 .
\end{array}
$$

There $\tau_{y}^{ \pm}$are the "tangent vectors" to the contour at the point $y$ (see fig. 1b), Kronecker symbols pick out the points (links) coinciding on the lattice and $C_{[x y]}$ is a part of the loop $C$ between the points $x$ and $y$, note that due to the presence of $\delta_{y x}$ it is also a closed contour. However, this notations, while being consistent, are too cumbersome. It is more convenient to use formally the continuum ones, i.e. to write the integrals instead of sums, delta functions instead of Kronecker symbols and so on. So we rewrite (2.4), simultaneously substituting the derivatives of $Z$ for the loop correlators according to (2.2), in the following form:

$$
\begin{aligned}
& L^{\mu}\left(C_{x}\right) Z=0 \\
& L^{\mu}\left(C_{x}\right)=\sum_{D} \beta_{D} \oint_{D} d y^{\mu} \delta(y-x) \frac{\partial}{\partial \beta_{C_{x} D_{y}}}+\oint_{C} d y^{\mu} \delta(y-x) \frac{\partial^{2}}{\partial \beta_{C_{[x y]}} \partial \beta_{C_{[y x]}}} .
\end{aligned}
$$


The system of equations (2.5) is overdefined, in the other words not all $L^{\mu}\left(C_{x}\right)$ are independent. One can verify that the operators (2.6) satisfy the following relations:

$$
L^{\mu}\left(C_{x}\right)=-L^{-\mu}\left(C_{x+\mu}\right) .
$$

If the marked link $l_{x}^{\mu}$ do not lie on the loop $C$ one can use the backtracking condition to give sense to this equation (see fig. 2). The second relation reads

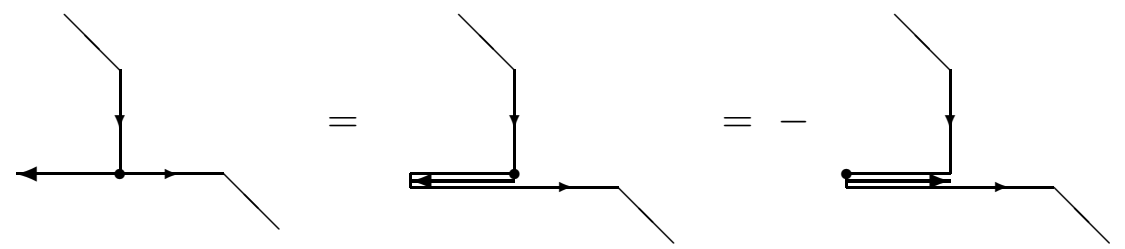

Figure 2: There we have used the backtracking condition (fig. 1a) and eq. (2.7).

$$
\sum_{\mu} L^{\mu}\left(C_{x}\right)=0 .
$$

Eq. (2.7) is a consequence of the unitarity of link variables and (2.8) is a manifestation of gauge invariance. Really, eq. (2.5) was obtained by an infinitesimal left shift of $U_{x}^{\mu}$, so the sum on the l.h.s. of (2.8) corresponds to the simultaneous left shifts of the link variables on all links originating from the point $x$. This is nothing that the gauge transformation.

For finite $N$ the additional constraints should be imposed on $Z$. These constraints follow from Mandelstam relations between multiple Wilson loops [6], which stems from the fact that $N \times N$ matrix has $N$ independent invariants. However, Mandelstam relations are invisible in the $1 / N$ expansion and one can abandon them, unless the nonperturbative effects (from the string theory point of view) are considered.

\section{The Loop Virasoro Algebra}

The eqs. (2.4) form a full set, i.e. they have a unique solution, at least within the perturbation theory. So the operators (2.6) should form a closed algebra with respect to commutation. Moreover, the calculation shows that this algebra, which is in what follows referred to as the Loop Virasoro Algebra (LVA), is linear, i.e. it's structure constants are $\beta$-independent $c$-numbers:

$$
\left[L^{\mu}\left(C_{x}\right), L^{\nu}\left(D_{y}\right)\right]=\oint_{C} d x^{\prime \nu} \delta\left(x^{\prime}-y\right) L^{\mu}\left(D_{y} C_{x^{\prime} x}\right)-\oint_{D} d y^{\prime \mu} \delta\left(y^{\prime}-x\right) L^{\nu}\left(C_{x} D_{y^{\prime} y}\right) .
$$

To find the basis in the LVA it is necessary to resolve the constraints (2.7) and (2.8). Eq. (2.7) shows that two operators corresponding to each link $l_{x}^{\mu}$ (with marked points placed at $x$ and at $x+\mu$ ) differ only by sign. Eq. (2.8) being a consequence of the gauge invariance, it's solution is equivalent to a gauge fixing. The standard way is to choose 
a maximal tree on the lattice and to put the gauge fields on all links belonging to this tree equal to unity. It is instructive to see how does this procedure look from the point of view of the solution of constraints (2.8). Let us resolve eq. (2.8) at some point $x$ for $L^{\mu}\left(C_{x}\right)$, consequently solve it at $x+\mu$ for $L^{\nu}\left(C_{x+\mu}\right)$ and so on. In this way one expresses all operators corresponding to the fixed links (i.e. that belonging to the gauge fixing tree) through $L^{\mu}\left(C_{x}\right)$ with $l_{x}^{\mu}$ being a non-fixed link. The latter operators form the basis of the LVA, because all the constraints will be resolved provided that the tree is maximal.

This gauge fixing has another important aspect. It determines a basis of generators of the loop space. Really, each loop can be represented as a product of the non-fixed links (we shall, however, distinguish the link $l_{x}^{\mu}$ and corresponding generator of the loop space, which we denote by $z_{x}^{\mu}$ ). If the end of some link in this representation does not coincide with the origin of the consequent link, one should connect them by a part of the gauge fixing tree (it can be always done due to it's maximality). It is evident that $z_{x}^{\mu}$ form a minimal set of generators.

As an example consider the one-plaquette (三 one-matrix) model. After the gauge fixing only one non-fixed link remains (see fig. 3). A loop in this model is characterized

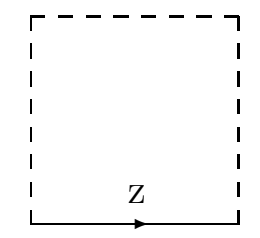

Figure 3: Gauge fixing in the one-plaquette model.

by it's winding number; in our notations the loop with winding number $n$ is denoted by $z^{n}$. The generators $L_{n} \equiv L\left(z^{n}\right)$ obey the commutation relations of the Virasoro algebra

$$
\left[L_{n}, L_{m}\right]=(n-m) L_{n+m} .
$$

It follows directly from (3.1) and reproduces the results of [ [4, 7]. If the plaquette is embedded in a more complicated lattice, one can associate with it the Virasoro subalgebra of the more wide LVA. More generally, each sublattice gives rise to the corresponding subalgebra in the LVA.

Now let us tern to a realization of the LVA, which may play an important role in it's string interpretation. This realization generalizes that of Virasoro algebra in terms of conformal transformations. As for the LVA, it can be interpreted as an algebra of infinitesimal changes of generators of the loop space:

$$
\begin{array}{r}
z_{x}^{\mu} \rightarrow z_{x}^{\mu}-\varepsilon C_{x} z_{x}^{\mu} \\
\left(z_{x}^{\mu}\right)^{-1} \rightarrow\left(z_{x}^{\mu}\right)^{-1}+\varepsilon\left(z_{x}^{\mu}\right)^{-1} C_{x} .
\end{array}
$$

To show it, let us introduce some definitions. First, we define the puncture operator creating a marked point on a loop:

$$
\hat{P}_{x} C_{x_{1} \ldots x_{n}}=C_{x x_{1} \ldots x_{n}}
$$


and derivative with respect to the $\operatorname{link} l_{x}^{\mu}$ operator, which acts on a loop according to the Leibnitz rule:

$$
\hat{p}_{x}^{\mu}=\oint d x^{\prime \mu} \delta\left(x^{\prime}-x\right) \hat{P}_{x^{\prime}}
$$

For the one-plaquette model $\hat{p}=z \frac{d}{d z}$; in the general case one can also formally treat $\hat{p}_{x}^{\mu}$ as a "differential" operator $z_{x}^{\mu} \frac{\partial}{\partial z_{x}^{\mu}}$. One can easily verify that the operators

$$
L^{\mu}\left(C_{x}\right)=-C_{x} \hat{p}_{x}^{\mu}
$$

obey the commutation relations of the LVA. The constraints (2.7) and (2.8) for (3.6) are the consequences of the obvious properties of derivative operators:

$$
\begin{gathered}
\hat{p}_{x}^{\mu}=-\hat{p}_{x+\mu}^{-\mu} \\
\sum_{\mu} \hat{p}_{x}^{\mu}=0 .
\end{gathered}
$$

After the gauge fixing, or, equivalently, the resolution of (3.7), (3.8), the operators (3.6) generates the transformations (3.3) while acting on the loops.

In the case of Virasoro algebra (3.6) reduces to the well known form of conformal generators:

$$
L_{n}=-z^{n+1} \frac{d}{d z}
$$

The analog of this formula existing for the LVA, it is interesting to understand what corresponds to the (classical) conformal fields. For spinless fields the answer is evident - consider a formal linear combination of loops with complex coefficients treating it as a function of generators $z_{x}^{\mu}$ :

$$
\Psi[z]=\sum_{C} \alpha(C) C
$$

The action of LVA on $\Psi[z]$ is given by (3.6). The spin- $k$ ( $k$-rank tensor) field can be defined as a set of formal linear combinations of the loops with $k$ marked points:

$$
\Psi_{x_{1} \ldots x_{k}}^{\mu_{1} \ldots \mu_{k}}[z]=\sum_{C} \oint_{C} d x_{1}^{\prime \mu_{1}} \delta\left(x_{1}^{\prime}-x_{1}\right) \ldots \oint_{C} d x_{k}^{\prime \mu_{k}} \delta\left(x_{k}^{\prime}-x_{k}\right) \alpha\left(C_{x_{1}^{\prime} \ldots x_{k}^{\prime}}\right) C_{x_{1}^{\prime} \ldots x_{k}^{\prime}}
$$

The LVA acts on (3.11) as the "Lie derivative":

$$
L^{\mu}\left(C_{x}\right) \Psi_{x_{1} \ldots x_{k}}^{\mu_{1} \ldots \mu_{k}}=-C_{x} \hat{p}_{x}^{\mu} \Psi_{x_{1} \ldots x_{k}}^{\mu_{1} \ldots \mu_{k}}-\sum_{s=1}^{k} \Psi_{x_{1} \ldots x_{s-1}}^{\mu_{1} \ldots \mu_{s-1}}\left(\hat{p}_{x_{s}}^{\mu_{s}} C_{x}\right)_{x x_{s+1} \ldots x_{k}}^{\mu \mu_{s+1} \ldots \mu_{k}} .
$$

Developing further the analogy with Virasoro algebra it would be interesting to find a free field representation of the LVA in terms of the objects like (3.10) or (3.11), i.e. to construct the generators $L^{\mu}\left(C_{x}\right)$ as the bilinear combinations of Fourier coefficients $\alpha(C)$ considered as the creation and annihilation operators. Then LVA may be regarded as a world-sheet symmetry of some "string theory". One may speculate that, analogously to the one-matrix model, $U(N)$ lattice gauge theory is equivalent to such "string theory" with zero dimensional target space. However, it is difficult to imagine what object plays the role of the world sheet in this theory, as the point on it is a set of noncommutative generators $z_{x}^{\mu}$ of the loop space. 


\section{Conclusion}

So far we have dealt with the lattice theory. The crossing to the continuum is a complicated and subtle procedure requiring an accurate tuning of the coupling constants. However, the main object of our interest, the LVA, is independent of the particular values of couplings, so the results of sec. 3 are valid in the continuum as well. One should only take seriously the continuum notations we have used. The treatment of the gauge invariance should also be slightly modified - instead of eq. (2.8) in the continuum the following relation is valid:

$$
\partial_{\mu}^{x} L^{\mu}\left(C_{x}\right)=0
$$

where $\partial_{\mu}^{x}$ is a path derivative (see [5] for a precise definition).

Thus the loop equations represents a wide symmetry algebra both in lattice and in continuum $U(N)$ gauge theory. One may hope that this symmetry displays the invariance of an underlying string theory. In this respect it would be interesting to relate our results with $1 / N$ expansion of the two dimensional QCD, which has more or less explicit string interpretation [3]. This problem deserves further investigation.

The author is grateful to L.Chekhov for discussions. The work was supported in part by RFFR grant No.93-011-147.

\section{References}

[1] K. Wilson, Phys. Rev. D10 (1974) 2445.

[2] V. Kazakov, A String Project in Multicolor QCD, the lectures given in the Trieste Spring School and Workshop-1993 on String Theory, hep-th/9308135;

I.K. Kostov, $U(N)$ Gauge Theory and Lattice Strings, talk delivered at the Workshop on string theory, gauge theory and quantum gravity, April 1993, Trieste, hepth/9308158.

[3] D. Gross, Nucl. Phys. B400 (1993) 161;

D. Gross and W. Taylor, Nucl. Phys. B400 (1993) 181; B403 (1993) 395.

[4] Yu. Makeenko, Mod. Phys. Lett. A6 (1991) 1901.

[5] A.A. Migdal, Phys. Rep. 102 (1983) 199.

[6] S. Mandelstam, Phys. Rev. D19 (1979) 2391.

[7] M. Bowick, A. Morozov and D. Shevitz, Nucl. Phys. B354 (1991) 574. 